\title{
Arthroscopy for treating temporomandibular joint disorders
}

\author{
Abstracted from \\ Rigon M, Pereira LM,BortoluzziMC, Loguercio AD, Ramos AL,Cardoso JR. \\ Arthroscopy for temporomandibular disorders. \\ Cochrane Database Syst Rev. 2011; 5: CD006385 \\ Address for correspondence: Luisa Fernandez Mauleffinch, Review Group Co-ordinator, \\ Cochrane Oral Health Group, MANDEC, School of Dentistry, University of Manchester, \\ Higher Cambridge Street, Manchester, M15 6FH, UK. E-mail: luisa.fernandez@manchester.ac.uk
}

\section{Question: Is arthroscopy effective for the management of signs and symptoms in patients with temporomandibular joint disorders (TMD)?}

Data sources The Cochrane Oral Health Group Trials Register, the Cochrane Central Register of Controlled Trials (CENTRAL), Medline Embase, Lilacs, Allied and Complementary Medicine Database (AMED) and CINAHL databases were searched. In addition the reference lists of the included articles were checked and 14 journals hand searched. Study selection Randomised controlled clinical trials (RCT) of arthroscopy for treating TMDs were included. There were no restrictions regarding the language or date of publication. Data extraction and synthesis Two review authors independently extracted data, and three review authors independently assessed the risk of bias of included trials. The authors of the selected articles were contacted for additional information. Pooling of trials was only attempted if at least two trials of comparable protocols, with the same conditions and similar outcome measurements were available. Statistical analysis was performed in accordance with the Cochrane Collaboration guidelines.

Results Seven RCTs $(n=349)$ met the inclusion criteria. All the studies were either at high or unclear risk of bias. Pain was evaluated after six months in two studies. No statistically significant differences were found between the arthroscopy versus nonsurgical groups (standardised mean difference $(S M D)=0.004 ; 95 \%$ confidence interval $(C l)-0.46$ to 0.55 , $\mathrm{P}=0.81)$. Two studies analysed pain 12 months after surgery (arthroscopy and arthrocentesis) in 81 patients. No statistically significant differences were found (mean difference $(\mathrm{MD})=0.10 ; 95 \% \mathrm{Cl}-1.46$ to $1.66, \mathrm{P}=0.90$ ). Three studies analysed the same outcome in patients who had been submitted to arthroscopic surgery or to open surgery and a statistically significant difference was found after 12 months $(\mathrm{SMD}=0.45 ; 95 \% \mathrm{Cl} 0.01$ to $0.89, \mathrm{P}=0.05)$ in favour of open surgery.

The two studies compared the maximum interincisal opening in six different clinical outcomes (interincisal opening over $35 \mathrm{~mm}$; maximum protrusion over $5 \mathrm{~mm}$; click; crepitation; tenderness on palpation in the TMJ and the jaw muscles 12 months after arthroscopy

This paper is based on a Cochrane Review published in the Cochrane Library 2011, issue 5 (see www.thecochranelibrary.com for information). Cochrane Reviews are regularly updated as new evidence emerges and in response to feedback, and the Cochrane Library should be consulted for the most recent version of the review. and open surgery). The outcome measures did not present statistically significant differences (odds ratio $(\mathrm{OR})=1.00 ; 95 \% \mathrm{Cl} 0.45$ to 2.21 , $P=1.00$ ). Two studies compared the maximum interincisal opening after 12 months of postsurgical follow-up. A statistically significant difference in favour of the arthroscopy group was observed $(\mathrm{MD}=5.28 ; 95 \% \mathrm{Cl} 3.46$ to $7.10, \mathrm{P}<0.0001)$.

The two studies compared the mandibular function after 12 months of follow-up with 40 patients evaluated. The outcome measure was mandibular functionality (MFIQ). This difference was not statistically significant ( $\mathrm{MD}=1.58 ; 95 \% \mathrm{Cl}-0.78$ to $3.94, \mathrm{P}=0.19$ ).

Conclusions Both arthroscopy and nonsurgical treatments reduced pain after six months. When compared with arthroscopy, open surgery was more effective at reducing pain after 12 months. Nevertheless, there were no differences in mandibular functionality or in other outcomes in clinical evaluations. Arthroscopy led to greater improvement in maximum interincisal opening after 12 months than arthrocentesis; however, there was no difference in pain.

\section{Commentary}

The question posed in this review is simple. Is arthroscopy effective in managing the signs and symptoms of temporomandibular joint disorders?

The review methodology is clearly set out though it is slightly disappointing to discover with all that has been written in relation to this subject only seven trials with 349 patients met the strict inclusion criteria.

The problem from a clinical perspective is that the variation of signs and symptoms in the presentation of temporomandibular joint pathology means that no study has compared like with like. The papers compare arthroscopy versus surgery, non surgical treatment, arthrocentesis and finally variations of arthroscopy technique.

Most surgeons would agree that the indications for open surgery and arthroscopy are different, but it is valid to compare different techniques and arthroscopy with the less invasive arthrocentesis.

Considering the surgical comparison, these were for chronic closed lock, but one group was treated with discectomy, another with a high condylar shave and the third with a combination of both of the above. This analysis found some benefit to the open surgery group, but the treatments are not comparable.

Comparing differing arthroscopic techniques is valid and this is mentioned in the review, but detailed figures are not produced for outcomes. 
Arthrocentesis is less invasive than arthroscopy and can be carried out in the out patient/office setting, hence the comparison is valid clinically and on health economic grounds. The results showed improved IID after 12 months in the arthroscopy group but no difference in pain scores (though these should have improved from baseline).

The review of non surgical treatment is interesting in that one study compared what we would consider as arthroscopy against surgery, occlusal rehabilitation and aggressive medical management. It is not clear how it was evaluated other than pain scores showing no difference. The second study only had 21 patients and a six month follow up and hence may not be truly representative.

The practical conclusions are valid in that for closed lock better IID is found at 12 months following arthroscopy rather than arthrocentesis. Also pain scores are lower following surgery. What is not mentioned is that the risk profile for surgery is much higher than for arthrocentesis or arthroscopy and that the former (and latter) can be easily repeated. Also the unstated inference has to be that the correct diagnosis been made before selecting a treatment. Correctly selected arthroscopy is invariably successful, poorly selected, eg degenerative joint disease, is rarely successful.

Finally the review highlights the paucity of good studies and the need for these to be carried out so more informed decisions can be made in treating these patients.

Roger Currie

Consultant Oral and Maxillofacial Surgeon, NHS Ayrshire and Arran and Hon Senior Clinical Lecturer University of Glasgow

Evidence-Based Dentistry (2011) 12, 90-91. doi:10.1038/sj.ebd.6400817 